doi:10.1017/\$104161022000352X

\section{Getting to know you but also to know me: changes in self-perceptions of aging among aged care workers after a Virtual Dementia Tour}

Virtual Dementia Tours (VDTs) afford an experience of what it might be like to live with dementia. VDT providers claim the experience has a range of benefits including more understanding and empathetic aged care staff, and better care for patients (e.g. Beville, 2002). Critical voices have also been raised, citing a weak evidence base for both the representation of the dementia experience (e.g. visual distortions that might better reflect the visual impairments experienced in medical conditions such as macular degeneration, but not dementia specifically) and the efficacy of the VDT experience in changing care (e.g. Merizzi, 2018). Importantly, reports of emotional distress following the VDT experience are worrying (Merizzi, 2018). To our knowledge, the specific impact of a VDT experience on attitudes toward one's own aging has not been researched. Our study (part of a larger analysis of the VDT; ethics approval \#2019001738) looked at how aged care workers' exposure to the VDT changed perceptions of their own aging.

A small group of aged care workers (3 males, 12 females; $M=39.75$ years old, $S D=10.18)$ including personal care attendants $(n=8)$ and nurses $(n=7)$ participated in the VDT as professional development training; all had worked in aged care for an average of just over 4 years. The VDT version delivered was based on Beville's original US-based study (2002), adapted for an Australian context (e.g. Australian coins used as stimuli). Prior to the VDT, participants completed demographic questions and the Reactions to Aging Questionnaire, designed to measure participants' beliefs about their own aging (RAQ; Gething, 1994).

During the VDT, participants wore items (such as yellow goggles and large gloves) to simulate aspects of aging (cataracts, loss of sensation, and fine motor skills) and were given confusing instructions and difficult to complete tasks while wearing headphones playing distracting noises (to simulate confusion and frustration as part of the experience of dementia). A short debrief offered an explanation about the VDT rationale and discussion of how participants reacted while in the experience.

A paired-samples $t$-test investigating the difference between pre- and post-VDT RAQ scores showed participants felt significantly more negative about their own aging after the VDT $(M=78.85$, $S D=23.23)$ compared with before $(M=96.21$, $S D=21.20) t(14)=2.43, p=0.029$ (see Figure 1), with a moderate effect size $(d=0.63)$.

The strength of this study was its novelty, being the first of its kind to investigate the effect of the VDT on participants' attitudes toward their own aging. Participants felt significantly more negative about their own aging following the VDT as measured by the RAQ. Previous research has found that negative exposure to aspects of aging leads to a more negative perception of aging. For example, Smith and Charlton (2020) found that growing up with an older adult in the home resulted in negative opinions of older adults years later, and that this was especially true if the older adult was ill. It could be that the VDT serves as an intense negative experience of dementia, which the contact hypothesis (Allport, 1954) would suggest is the mechanism for this change in feelings about aging.

Our data suggest a critical role of debriefing, not simply for reflections on living with dementia, but also about how such an experience affects personal views about aging. The VDT highlights sensory, motor, and cognitive changes underscoring how difficult it is to navigate spaces and complete tasks with dementia. However, the experience of shifting from a sensory and cognitive experience of functioning as a person without dementia, to a person who has suddenly lost these capacities, can be a profoundly negative experience, as other authors (e.g. Merizzi, 2018) have noted. The debriefs employed here focused on explaining the mapping of the VDT onto the experience of a person living with dementia, rather than the person's own sensemaking of the experience, as someone who themselves is aging (as all people are).

It is recommended that participants be informed of the potential emotional effects of the VDT and were asked to consider whether the experience is suitable for them. A more comprehensive debrief is also recommended, where participants can reflect on their own experience as a caregiver and an aging person. There should be discussion around limitations of the VDT itself, where participants are informed that dementia is not solely characterized by continuous negative experiences, and that not all older adults will experience dementia. These changes could minimize distress and negative views about aging reported following the VDT. 


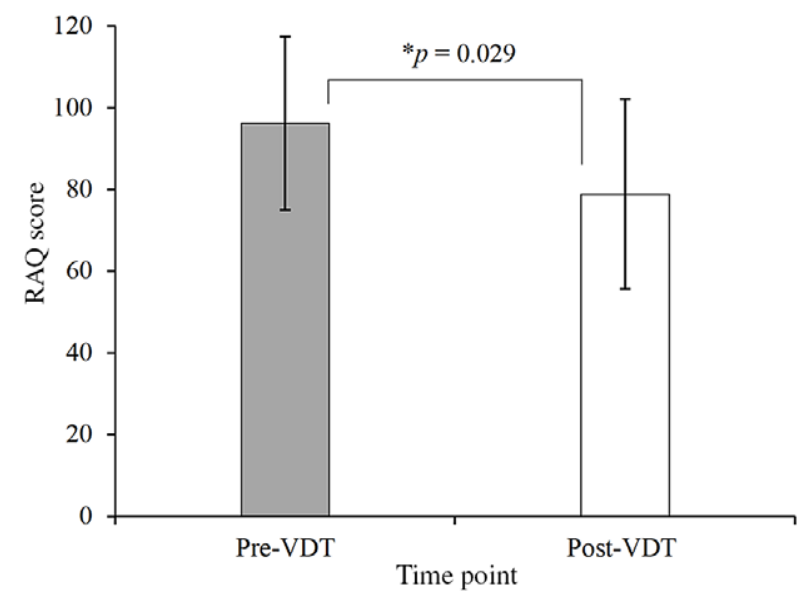

Figure 1. Pre- and post-VDT scores on the RAQ. Error bars represent standard deviations.

\section{References}

Allport, G. (1954). The Nature of Prejudice. Cambridge, MA: Addison-Wesley.

Beville, P. K. (2002). Virtual dementia tour ${ }^{\mathcal{O}}$ helps Senditiz health care providers. American fournal of Alzheimer's Disease E Other Dementias, 17, 183-190.
Gething, L. (1994). Health professional attitudes towards ageing and older people: preliminary report of the Reactions to Ageing Questionnaire. Australian fournal on Ageing, 13, 77-81.

Merizzi, A. (2018). Virtual dementia tour ${ }^{\circledR}$ : limitations and ethics. Quality in Ageing and Older Adults. https://doi.org/10 .1108/QAOA-11-2017-0042

Smith, B. T. and Charlton, K. (2020). When grandparents move in: consequences of growing up with older adults. Social Psychology. https://doi.org/10.1027/1864-9335/ a000415

Lana Farina, Jordan Asnicar, Olivia H. Chan and Nancy A. Pachana (i)

School of Psychology, The University of Queensland, Brisbane, QLD, Australia

Correspondence should be addressed to: Professor Nancy A. Pachana, School of Psychology, The University of Queensland, Brisbane, Qld, Australia, tel +61733 656832; fax +61733654466. Email: n.pachana@psy.uq .edu.au 